\title{
Spike-Nosed Bodies and Forward Injected Jets in Supersonic Flow
}

M. Gilinsky, C. Washington Hampton University, Hampton, VA 23668

I.M. Blankson

NASA Glenn Research Center, Cleveland, OH 441325

\author{
and \\ A.I. Shvets \\ Institute of Mechanics of Moscow State University \\ Moscow, 117192, Russia
}

\section{$3^{\text {th }}$ AIAA/ASME/SAE/ASEE Joint Propulsion Conference and Exhibit 7-10 July 2002/Indianapolis, Indiana}




\title{
Spike-Nosed Bodies and Forward Injected Jets in Supersonic Flow
}

\author{
M. Gilinsky", C. Washington ${ }^{* *}$ \\ Hampton University, USA \\ I.M. Blankson \\ NASA Glenn Research Center, USA \\ and \\ A.I. Shvets**** \\ Institute of Mechanics/Moscow State University, Russia
}

\begin{abstract}
The paper contains new numerical simulation and experimental test results of blunt body drag reduction using thin spikes mounted in front of a body and one- or two-phase jets injected against a supersonic flow. Numerical simulations utilizing the NASA CFL3D code were conducted at the Hampton University Fluid Mechanics and Acoustics Laboratory (FM\&AL) and experimental tests were conducted using the facilities of the IM/MSU Aeromechanics and Gas Dynamics Laboratory. Previous results were presented at the $37^{\text {th }}$ AIAA/ASME/SAE/ ASEE Joint Propulsion Conference [1]. Those results were based on some experimental and numerical simulation tests for supersonic flow around spike-nosed or shell-nosed bodies, and numerical simulations were conducted only for a single spike-nosed or shell-nosed body at zero attack angle, $\alpha=0^{\circ}$. In this paper, experimental test results of gas, liquid and solid particle jet injection against a supersonic flow are presented. In addition, numerical simulation results for supersonic flow around a multiple spike-nosed body with non-zero attack angles and with a gas and solid particle forward jet injection are included. Aerodynamic coefficients: drag, $C_{D}$, lift, $C_{L}$ and longitudinal momentum, $\mathrm{M}_{\mathrm{Z}}$, obtained by mumerical simulation and experimental tests are compared and show good agreement.
\end{abstract}

1. Preview. A thin spike mounted in front of a blunt body can be used to decrease the drag and the heat transfer in a high oncoming flow velocity. By varying the length of the protruding spike, the aerodynamic characteristics of the apparatus can be controlled; a thin straight spike is a convenient and simple means of control. A summary of the early experimental and theoretical papers where flow past a body with a spike was studied is contained in the well-known book by P.K. Chang (1970) and in the Russian book [2].

Six regimes of supersonic flow past a cylinder with conical nose and a spike protruding from it are distinguished for

\footnotetext{
* Research Professor, Senior Member AIAA

** Assistant Professor

*** Senior Scientist, AIAA Associate Fellow **** MSU Professor, IM/MSU Lab. Chief, Russia
}

specified Mach and Reynolds numbers of the oncoming flow which include the regime of stationary flow with drag reduction and the regimes of unsteady pulsating and fluctuating flows. A short review of different experimental and numerical simulation approaches for the analysis of this problem was presented in the paper [1]. A detailed review of this area is in preparation at the present time and will published as a NASA GRC report soon.

\section{Experimental Test Results. Spike-Nosed Blunt Bodies \\ Experimental test results [1] were obtained using an axisymmetic cylindrical model with a flat forward part, i.e. butt-end, of diameter, $\mathrm{D}=80 \mathrm{~mm}$. Two types of models were tested: a) with 1.needle $(\mathrm{n}=1)$ and $\mathrm{b})$ with 5 needles $(n=5)$. Several such designs are shown in}


Figure 1. In both cases, one central needle was mounted at the center of the circular front flat part of radius, $R=40 \mathrm{~mm}$

For the second series of models, another 4 needles were placed symmetrically around the central needle. They were placed at the angular interval of $90^{\circ}$ and at the radial distance, $r=0.5 \mathrm{R}$. Non-dimensional needle lengths, L/D, were chosen based on constructive opportunities of application, and on known referenced data in this field. These data show that maximum drag reduction using needles at supersonic speeds can be achieved in the range of needle lengths, $\mathrm{L} / \mathrm{D}=1.5-2.0$, so that three needle lengths were manufactured and tested: $\mathrm{L} / \mathrm{D}=1.5,1.0$, and 0.5. For this needle length range, the end of the needle at one limit, $L / D=1.5$, is located farther from the butt-end than the detached bow shock wave which occurred in the case of flow without a needle at the body front. For the other limit, $L / D=0.5$, the needle is located completely inside the compression shock layer, i.e. closer to the butt-end than the corresponding detached bow shock wave. As a result of this analysis, the following needle geometric parameters were chosen: $D / L=0.05 ; 0.81 ; 0.1$, i.e. for the given model size, the needle diameters are: $d=4 ; 6.5$; and $8 \mathrm{~mm}$.

Optical methods of flow visualization were employed for better understanding of this phenomenon and for explanation of the aerodynamic characteristic changes. They include a traditional schlieren method and high resolution video filming of the unsteady pulsation regimes. In Figure 1, the main models tested in supersonic flow with Mach number, $M=3$, at zero angle of attack are shown. From the left to the right, the first row: 1)-butt-end without needles; 2)-4) -5 thin needles model of thickness, $d / D=0.05$, and different needle lengths, $L / D=0.5,1.0$ and 1.5. In the second row: 5)-7) -with a single needle for two different instants of the pulsation flow regime.

Several additional experimental tests were conducted to determine the influence of multi-needle $(n>5)$ and shell applications to the force coefficients. The needle numbers were: $n=53$ and $n=103$. These needles are mounted symmetrically along coincident circles located from the circle center on the radial distances: $R=0.5-35 \mathrm{~mm}$. Recall that the butt-end circle diameter is $D=80 \mathrm{~mm}$. A drag coefficient comparison for all considered cases, $\mathrm{n}=1 ; 5 ; 53$ and 103 illustrates a preferable application for drag reduction of single needles.

The principal conclusions of these test results for spikes were: multiple spike/needle application leads to decrease of drag reduction benefits by comparison with the case of one central mounted needle at the front of a blunt body, as well as in longitudinal moment, but an increase in lift benefits. Dependence of the butt-end drag coefficient, $C_{D}$; of relative needle length, $\mathrm{L} / \mathrm{D}$, and relative needle diameter, $\mathrm{r} / \mathrm{D}$, is shown in Figure 2. Here D is the butt-end diameter; free stream Mach number, $M=3$, and angle of attack, $\alpha=0^{\circ}$.

\section{Numerical Simulation Test Results: Spike-Nosed Blunt Bodies}

Numerical simulation tests for the 1-needle model were conducted using the IM/MSU Russian code, and the main results were also presented at the previous propulsion conference [1]. This code is based on the Navier-Stokes equations for modeling of gasdynamic and chemical processes taking place in internal as well as external gas flows. These results were obtained using a laminar viscous gas model as well as the $k-\omega$ turbulence model. It was observed that the flow regime around the spike-nosed blunt body obtained in numerical simulation is 
very sensitive to changes of the numerical scheme parameters or applied models. For example, for the same conditions in inviscid flow (Euler approximation) or for a laminar viscous flow, numerical simulation results show periodic oscillatory variation of the flow. Such observations were found in [1] for the case: free stream Mach number, $M_{\infty}=3$, $\mathrm{L} / \mathrm{D}=1$, pressure, $\mathrm{P}_{\infty}=0.14$, temperature, $\mathrm{T}_{\infty}$ $=102^{\circ} \mathrm{K}$, and Reynolds number, $\mathrm{Re}_{\infty}$ $=1.8 \times 10^{6}$. However, application of the $\mathrm{k}-\omega$ turbulence model has led to stabilization of this process for the same outer boundary conditions in agreement with experimental test results known from our own and several other published papers.

Numerical simulation tests of a 2D supersonic flow for the 2-step configuration have been conducted for different free stream Mach numbers, $M=1.5-4.0$ and different lengths of forward directed small height steps. This 2D problem is analogous to the problem of supersonic flow around a 2needle model described above in the previous section. Accurate solution of this problem allows resolution of many obstacles connected with the more complicated 3Dproblem solution for the flow in the 5needles model.

New numerical simulation results were obtained for the 3D problem using the NASA CFL3d code [3] for the case of zero attack angle, $\alpha=0$, as well as non-zero attack angle in the range of: $-15^{\circ} \leq \alpha \leq+15^{\circ}$. Some information for supersonic flow numerical simulation with free stream Mach number, $\mathrm{M}_{\infty}$, around a 5-needle nosed butt-end at zero angle of attack was presented at the conference [4]. The NASA CFL3D code was employed, and Navier-Stokes Equation (NSE) based simulations were conducted with slip and no-slip boundary conditions at the solid walls. An example of such results is illustrated in Figure 3. Presented there are: a)
Mach contours in the symmetry XZ-plane; $b$ ) Mach contours in the cross section $X=$ const at the butt-end front wall; c) a portion of grid employed in this cross section; d) 3D grid for the domain between two symmetry planes. This grid contains 15 blocks. In this case, slip boundary conditions were applied. Note that numerical simulation results are in good agreement with experimental test results obtained in this research.

\section{Liquid Jet Injection from the Blunt Body Front against a Supersonic Flow}

\subsection{Technique of Experiments. The} structure of interaction of liquid jets exhausted towards to subsonic or supersonic airflow has been considered. Two types of interaction: were analyzed: injection from a conical divergent ledge with an exposure angle, $\alpha=120^{\circ}$, installed at the blunt body front and injection directly from the flat part of butt-end. In the latter case, the nozzle was installed at the end of the cylindrical divergent tube of diameter, $0.1 \mathrm{D}(8 \mathrm{~mm})$, and wall thickness varied from 0.2 to $0.5 \mathrm{~mm}$. This tube was strengthened at the center of the flat part butt-end. The tested models had the following geometric characteristics: buttend diameter, $D=80 \mathrm{~mm}$, and nozzle exit diameter was varied discretely: $d=0,34$; 0,$64 ; 1,48 ; 2,8 ; 3 ; 4$; and $5 \mathrm{~mm}$. It is known that liquid jet injection from a nozzle can be accompanied by cavitation. To avoid cavitation, the nozzle had a smooth longitudinal shape of the input portion extended by a cylindrical tube of various lengths from 80 to 150 nozzle diameters.

Experimental tests were conducted in the wind tunnel, A-7, and in the special jet stand, both located at the Institute of Mechanics of Moscow State University. The test chamber of the wind tumel has square cross section, $0.6 \times 0.6 \mathrm{~m}$. Free stream Mach number, $M$, varies from $M=0.3$ to $M=3$, and Reynolds 
number, $R e$, varies from $1 \times 10^{6}$ to $3 \times 10^{7}$ calculated using free stream parameters and the characteristic length, $1=1 \mathrm{~m}$. During experimental tests, schlieren photos of the flow field were made and the main free stream and injected liquid jet parameters were measured. Also, the pressure distribution along the butt-end radial direction was measured as well as at the nozzle exit and inside of the separation zone. The average velocity value of the liquid jet exhausted was calculated from the liquid mass flow rate. The latter was determined using pressure difference at two sizes of the special measured washers. The relative rootmean-square error of velocity measurements of a liquid was estimated as \pm 0.05 .

\subsection{Experimental Test Results:} Geometrical Characteristics. In Figure $4 a, b$, a shlieren picture of a liquid jet interacting with a supersonic airflow is shown for two cases. In a) the jet exhausted from a conic body against the flow with zero angle of attack; b) the jet exhausted from a flat forward part of the butt-end with zero angle of attack. Dependence of butt-end drag coefficient, $C_{D}$, vs the relative specific mass flow rate of a liquid jet, $G=\rho_{j} U_{j} / \rho_{\infty} U_{\infty}$, and the relative specific impulse $\mathrm{K}=\rho_{\mathrm{j}} \mathrm{U}_{\mathrm{j}}{ }^{2} / \rho_{\infty} \mathrm{U}_{\infty}{ }^{2}$ are shown in Figure 4c.

Four potential schemes of supersonic airflow interaction with liquid jet exhausted from a conical nozzle and butt-end were observed in the experimental test series. These schemes are illustrated in Figure 5. For moderate speeds of the liquid jet exhausted from the conical nozzle (Figure 5a,b), the jet has almost a cylindrical-shaped portion, 4 , and then the jet sharply transforms to almost spherical shape, 6, as shown in Figure 5a or almost mushroom-shape as shown in Figure 5b. Further downstream, these jets break up to multiple droplets. This area of the flow represents a complicated mixture of liquid droplets and bubbles. At the blunt jet portion, the bow shock wave, 7 , is formed. Also, a separation zone 3 is formed between the conical and cylindrical liquid jet portions. In the separation zone, static pressure was measured using the special pressure transducer, 2.

The structural picture of liquid jet interaction with the main air stream depends on the nozzle shape and relative jet diameter. If the jet penetration depth is much more than the nozzle diameter, the liquid jet interacting with the air stream is close to stationary. Photos of such interaction are shown above in Figure 4a. In the presented experimental tests, fluctuations of jet penetration depth were observed for some regimes. Therefore the visualized shock waves are a little bit dim because of the relatively large exposure 0.01 employed.

It was observed that in the case of liquid jet injection a stationary regime of interaction takes place only for relatively small mass flow rate of the injected jet. In this case, the jet does not penetrate the bow shock wave formed at the blunt body without injection (Figure 4a). Otherwise, an unsteady oscillatory regime takes place (Figure 4b). The same effect was observed in the case of solid needle application noted in [1]. However, for liquid jets, this effect is more sensitive to the boundary conditions producing strong interaction of two deformation media-a gas and liquid. At large liquid mass flow rates, there are intensive fluctuations of the bow shock wave. Shock wave fluctuations are a cause of liquid mass flow rate fluctuations at the body front etc. The schematic picture of such interaction is illustrated in Figure 4d. Formation of protuberances emitted through an external jet confirms that the main jet portion experienced intensive cross fluctuations connected with fluctuations in the jet mass 
flow rate. In the non-stationary interaction regimes, static pressure along the butt-end face was essentially reduced (on $70 \%-80$ $\%$, relative to pressure behind the normal portion of the bow shock wave, a distinctive analogue with a hydraulic jump.

Geometric characteristics of a liquid jet exhausted from a conical nozzle and butt-end obtained in experimental tests are presented in Figure 6. In this figure, dependence of relative jet penetration depth, $\varepsilon$, vs root of a relative jet impulse $\sqrt{\mathrm{K}}$ is shown. Here are $\varepsilon=1 / r$, where 1 is jet penetration depth and $r=$ nozzle radius. Points 1-3 correspond to the jets with diameters: $d=3,4,5 \mathrm{~mm}$, flowing from a conical nozzle into the airflow with Mach number, $M=3$. The point 4 corresponds to the jet flowing from butt-end hole with $\mathrm{d}=3 \mathrm{~mm}$. One can see that, in the case of a stationary regime $(K<D)$ when the jet does not interaction with a shock wave, the jet penetration depth surpasses the appropriate depth for a free jet. In contrary case $(K>1)$, when interaction between shock waves takes place, jet penetration depth becomes less than the corresponding value for the free jet flowing to a still space. However, there is monotone behavior of jet penetration depth vs $K$ even with change of the interaction regime. Note that, in regimes of nonstationary interaction, the fastest destruction of the liquid jet takes place in the vicinity of the blunt body.

Liquid jets injected from the conical forward part of the body interact with a contrary gas stream by formation of a forward thickening drop-shaped structure. This occurs for a wide range of characteristic parameters with ringshaped returnable gas-fluid jet downstream at the body periphery. The external boundary of the returnable ring jet can be described as a conical surface with good accuracy. Thus, the form of the returnable jet can be characterized by two parameters: conical angle, $\varphi$ and external diameter $\mathrm{D}_{c}$ of the returnable jet in the plane of the nozzle exit. Results of measurements are shown Figure 7, where dependence on angle, $\phi$, and relative returnable jet diameter, $d_{c}=D_{c} / d$, are shown. Practically, these values do not depend on free stream Mach number in the region: $\mathbf{M}=$ $0,3-1,2 ; 2 ; 3$. They are defined by the parameter $\sqrt{\mathrm{K}}$ as well as by the relative jet penetration depth, $\varepsilon$. In this figure, points 1-3 are for $d_{c}=f(\varepsilon), d=3,4,5 \mathrm{~mm}$, and 4-6 are for $\varphi=f(\varepsilon), d=3,4,5 \mathrm{~mm}$. Experimental data are approximated using the dependence $\varphi=5 / 3 \pi$ $/ \varepsilon+4, d_{c}=3.6 \varepsilon^{1 / 3}$

Based on the conducted experiments, some dependence between the diameter of the return jet and diameter of the butt-end was observed. So, for example, it is possible to predict the transition from stationary to nonstationary regimes of interaction. For relatively small jet penetration depths, which do not surpass the shock layer thickness (without a jet) at the butt-end, isolated jet stationary regimes can be predicted. With increase of jet speed accompanying jet penetration, whole shock layer, nonstationary modes of interaction can be predicted. The experimental data show that non-stationary regimes take place until the characteristic size satisfies the inequality, $d_{c}$ $<d_{r}$, where $d_{r}=D_{r} / d$. A further increase of speed leads to the stationary interaction regime, in which the returnable jet does not reach the front face platform of the blunt body.

\subsection{Blunt body drag coefficient variation} with liquid jet injection. Various methods were employed for the purpose of blunted bodies drag reduction. These methods favor the formation of separation zones at the blunt body face. As a result, a detached shock wave transforms to a wave similar to the shock wave arising at the conical or ogivalshaped body in a contrary supersonic flow. 
With the purpose of formation of such separation zones, gas or liquid jet injection may be applied as well as firm particle emission alongside with widely investigated methods of solid needle application

A special construction was manufactured for more accurate drag measurements. The model was a cylindrical body, with a flat forward part. A flexible hose was attached to the body with the device for measuring the reduction of force. Calibration of the tension-balance was carried out at various pressures of the flow system. On the forward part of the cylinder, a rifled hole was made for installation of nozzles of various diameters.

Experimental tests were carried out with a cylindrical model of small length $(L=D, D=$ $80 \mathrm{~mm}$.) with both flat and hemispherical head parts. The liquid flowed from a cylindrical nozzle. The internal nozzle diameters were 4 and $2 \mathrm{~mm}$ and the nozzle length was equal to $8 \mathrm{~mm}$. The first tests have shown that water jet injection from the nozzle towards a supersonic stream with Mach number, $M=3.0$, leads to freezing of the liquid and transferring to ice at some distance from the nozzle. Therefore in these experiments, an anti-freezing liquid was used, represented by the mixture of ethyleneglycol (30-50 \%) and water (70 - $50 \%)$. Density of this liquid, $\gamma=0.8-1.05$.

The efficiency of influence of liquid jet injection was examined for free stream Mach number, $M=3$. In Figure 8, the dependence of the drag coefficient, $C_{D}$, of a cylindrical body with flat and spherical forward parts vs parameter $\sqrt{\mathrm{K}}$ of the liquid jet is illustrated. In both cases, this jet was exhausted from a nozzle with the same relative diameter, $d / D$. Figure 6 illustrates that jet injection leads to monotonic body drag reduction with increase of parameter $\sqrt{\mathrm{K}}$. These results have also shown that the value of reduction of aerodynamic drag of the model is defined not only by the mass flow rate of the liquid jet, but also by jet diameter. For each nozzle size, there is a size relative to the mass flow rate at which a drag coefficient, $C_{D}$ is minimal. A further increase of the jet mass flow rate leads to growth in the drag coefficient, $C_{D}$.

For solid particle injection, a nozzle of diameter, $\mathrm{D}_{\mathrm{n}} / \mathrm{D}=0.1$, was manufactured. Appropriate schlieren photos of the flows have shown that injected particles smash and destroy the bow shock waves detached at the butt-end model. It is observed that particle injection leads to butt-end drag reduction, then the drag coefficient increases. Comparison of these experimental test results with numerical simulation results will be presented in the next section.

\section{Numerical simulation test results: Solid Particles}

An existing discrete-trajectory approach using the two-phase version of the $1^{\text {st }}$ order Godunov scheme for numerical simulation of unsteady and steady 2D and axisymmetric inviscid flows [5] was improved. The improvement lay in two directions: 1) use of "almost adaptive" grids for gas phase and 2) application of a $2^{\text {nd }}$ order numerical scheme for gas phase and calculation of particle trajectories and their parameters along these trajectories. By such improvements, more exact numerical results were obtained for the problem of supersonic flow at the blunt body with solid particle injection against a flow. Several numerical simulations were conducted for the cases tested experimentally in the $\mathrm{IM} / \mathrm{MSU}$ aerodynamic wind tunnel A7. At the present time, we are developing the discrete-trajectory method for the NASA CFL3d code for numerical simulation of twophase flows based on the NSE approximation for gas phase. 
This method has been used to determine sensitivity of the wave drag of a cylinder and face (with the radius $r *$ to several parameters. In particular, a) the mass flow rate $\varepsilon$ of the particles in the injected mixture, b) the particle size $a_{p}$, and c) the particle slip ratio $l_{p}=\mathfrak{u}_{p w} / \mathfrak{u}_{w}$ (with respect to the carrier gas velocity) at the injector exit section (of radius $\left.y_{w}\right)$.

The flow conditions are as follows:

$\mathbf{k}_{\infty}=\mathrm{k}_{\mathrm{w}}=1.4: M_{\infty}=3 ; \mathrm{M}_{\mathrm{w}}=1.05$;

$\mathrm{y}_{w} / \mathrm{r}_{\theta}=0.1 ; \mathrm{p}_{\text {ow }} / \mathrm{p}_{\infty}=0.67 ; \mathrm{T}_{\text {ow }} / \mathrm{T}_{\infty}=1$;

$\mathrm{T}_{\mathrm{p}} / \mathrm{T}_{\mathrm{w}}=1 ; \quad \beta=0.014 ; \gamma=4.8 .10^{-6}$;

Re. $=1.48 \cdot 10^{4}\left(a_{p}=1 \mu k m\right)$.

Parameters of the particles varied in the following ranges: $0<a_{\mathrm{p}}<1 \mu \mathrm{km}, 0<\varepsilon<1$, $0<\mathrm{L}_{\mathrm{w}}<1$, where $\mathrm{L}_{\mathrm{w}}=U_{w} / U_{\infty}$. Used as reference values are free-stream parameters for the stagnation point and the cylinder radius. In the present analysis, two relative values are essential, the jet gas mass flow rate $G_{w}=\rho_{w} u_{w} / \rho_{\infty} v_{\infty}=2.84 ;$ and the momentum $\quad K_{w}=\rho_{w} u^{2}{ }_{w} / \rho_{\infty} v_{\infty}^{2}=1.49$; Non-dimensional parameters, $\beta$ and $\gamma$ characterize force interaction and heat transfer between single particle and carrying gas. Reynolds number, $R_{*}$, is calculated using gas critical (sound) parameters and characteristic length, $1 \mu \mathrm{m}$.

Figure 9 demonstrates location of a bow shock wave depending on the particle size, $\mathrm{a}_{\mathrm{p}}$, and the mass flow rate of particles, $\varepsilon$. It is readily seen that particles introduced in the jet do enhance the distance between the shock wave and the body, the influence of the particle size being more notable than that of the particle mass flow rate. Particles represented by the shaded area in the figure (at $\varepsilon=0.3, \mathrm{a}_{\mathrm{p}}=1 \mathrm{~mm}$ ) move along the symmetry axis to a surface where horizontal components of their velocities become zero. After being turned, they are in the circulation return flow and are moved as a thin layer by the incident flow. This layer seems to be describable by the term "sheet" [6].

Figure 10 contains the function $\mathrm{C}_{\mathrm{xo}}(\varepsilon)$ for the case with particles of size $a_{p}=1 \mu \mathrm{km}$ (line1). The line 6 represents the distance $x_{p}$ $(\varepsilon)$ attained by the particle in their backpenetration along the symmetry axis. Here, this distance is roughly the same as the maximum separation of the shock wave and as the length of the circulation return flow. region. The rate of the decrease in $\mathrm{C}_{\mathrm{xo}}$ is the greatest, as great $12 \%$, at $\varepsilon=0.05,23 \%$. Thus, we conclude that even a minor amount of particles leads to a noticeable reduction in the wave drag. This same figure contains the numerically obtained function $\mathrm{C}_{\mathrm{xo}}(\varepsilon)$ for a body with a needle of length $1=x_{p}$ (dot-anddash line 4 , for an "effective" needle). Injecting a heterogeneous jet is seen in curve 5 for a non-monotonous function $\mathrm{C}_{\mathrm{xo}}\left(\mathrm{a}_{\mathrm{p}}\right)$ which attains its maximum at $a_{p}-0.5 \mu \mathrm{km}$ $\left(\varepsilon_{0}=0.3\right)$. The dashed line 3 is for experiments involving bodies with needles of length $1=x_{p}$. As long as $a_{p}<1.5 \mathrm{~mm}$, convergence of results is satisfactory. When $a_{p}>1.5 \mu \mathrm{km}$, the practical back-penetration distance (curve 2) and the circulation return flow region length monotonously increase in $a_{p}$, and $c_{x} 0$ continues to decrease. However, the experiment performed for needle lengths $1>5 \mathrm{r}$ * reveals an abrupt increase in $\mathrm{C}_{\mathrm{xo}}$ due to the flow separation onset being shifted from the needle tip toward the body (the so-called delayed separation). Computation does not predict this effect.

The numerical result leads to the conclusion that large particles $\left(a_{p}>a_{p^{*}}\right)$ are preferable to small ones. However, this is not so in the general case. The reality is that particles at the nozzle exit section are slower than the carrier gas. The minimum value of $c_{x} 0$ on the curve 1 in fig. 12 is at $L_{w}=1$ when the particles fly the maximum distance (curve 2) 
and decelerate least near the nozzle. The dashed line 3 corresponds to the "effective needle", $1=x_{p}$. When $L_{w}<0.5$, the coefficient $\mathrm{C}_{\mathrm{xo}}$ is greater than $\mathrm{C}_{\mathrm{xo}}^{0}$, so that a pure gas jet is more advantageous. With due account for the reaction force, the effective drag coefficient

$C_{x e}=\frac{F}{\pi r_{\infty}^{2} \rho_{\infty} v_{\infty}^{2} / 2}=C_{x 0}+2\left[\frac{y_{\infty}}{r_{*}}\right] K_{w}\left(1+\varepsilon L_{w}\right)$

is in notable excess over the $\mathrm{C}_{\mathrm{xo}}$ only if the particle mass flow rate is great enough. The functions $\mathrm{C}_{\mathrm{x}}=\mathrm{C}_{\mathrm{xo}}(\varepsilon)$ and $\mathrm{C}_{\mathrm{x}}=\mathrm{C}_{\mathrm{xe}}(\varepsilon)$ are plotted as curves 4 and 5 , respectively.

Calculation shows that the extent of decrease in drag depends on the distance from the body along the symmetry axis to the point where the particles stop (i.e. depends on the "two-phase" cone apex angle). One of the likely options for shifting this point is to supply the heterogeneous jet through a needle nozzle of length 1 . How $\mathrm{C}_{\mathrm{xo}}$ depends of $l_{p}=1 / r *$ is represented in Figure 11 by curves 6 and 7 for $\varepsilon=0$ and $\varepsilon=0.3$, respectively. The "needle + jet" combination definitely causes $\mathrm{C}_{\mathrm{x} 0}$ to further decrease. For example, if $l_{p}=2$, the drag is reduced by a factor of approximately two. No oscillation in a flow with the mentioned parameter is predicted.

Finally, in Figure 12, a comparison of numerical simulation and experimental data is shown for different sizes of monodispersion quartz particle jets interacting with the air flow with Mach number, $M=3$. The experimental tests were conducted at the IM/MSU supersonic wind tunnel, A-7. This comparison demonstrates a satisfactory agreement between the theory and experiment.

\section{Conclusion}

New numerical simulation and experimental test results on blunt body drag reduction due to thin spikes mounted in front of a body and one or two-phase jets injected into a supersonic flow have been presented. The experimental and numerical results are in good agreement and show that significant drag reduction can be achieved by proper choice of spike and flow parameters. Beneficial parameter ranges are described.

\section{Acknowledgements}

We would like to acknowledge the NASA Glenn Research Center, especially Mr. Robert C. Hendricks for initiation, interest and support of our research. This research was partially conducted under the NASA grant NAG-3-2422 and under the supporting CRDF grant RE1-2068. We would like to thanks Dr. Jay C. Hardin for his attention, interest in our research and useful suggestions.

\section{References}

1. Gilinsky, M., Blankson, I.M., Sakharov V.I., Shvets, A.I., 2001, Shock Waves Mitigation at Blunt Bodies using Needles and Shells against a Supersonic Flow, 37 $7^{\text {th }}$ AIAA/ASME/SAE/ASEE Joint Propulsion Conference, 8-11 July, 2001, Salt Lake City, UT, USA, 12p.

2. Shvets A.I., Aerodynamics of Supersonic Shapes, 1987, Moscow State University Press, Moscow.

3. Krist, S.L., Biedron, R.T., and Rumsey, C.L., 1996, CFL3D User's Manual (Version 5.0), NASA Langley Research Center, 311p.

4. Gilinsky, M., Patel, K., Alexander, C., at al., 2001, Numerical Simulation of One- and TwoPhase Flows in Propulsion Systems, HBCUs/OMUs Research Conference Agenda and Abstracts, OAINASA GRC, April 17-18, 2001, p.23.

5. Gilinsky, M.M., et al., 1994, Supersonic Gasdispersional Jets: Models and Applications, AIAA Paper 94-0135, 32nd Aerospace Sciences Meeting and Exhibit, Jan. 10-13, 1994/Reno, NV.

6. Krayko, A.N., 1982, To two-liquid model of a gas flow with dispersion particles, Applied Mathematics and Mechanics, V.6, No 1, pp. 96106 

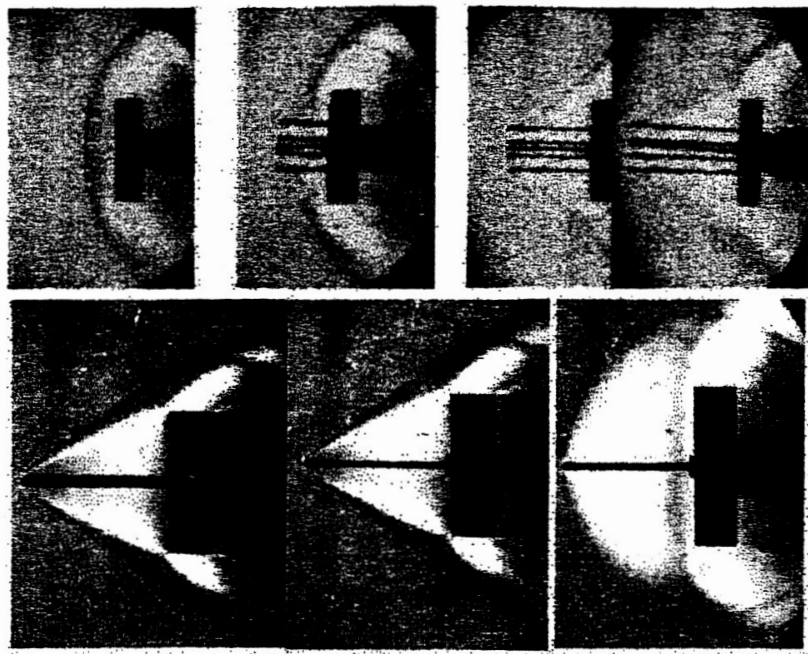

Fig. 1 Interaction of supersonic air flow with spike-nosed blunt body having 1 and 5 needles. Experimental test results. Shlieren photos. Free Stream Mach number, $M=3$, angle of attack, $\alpha=0^{\circ}$.

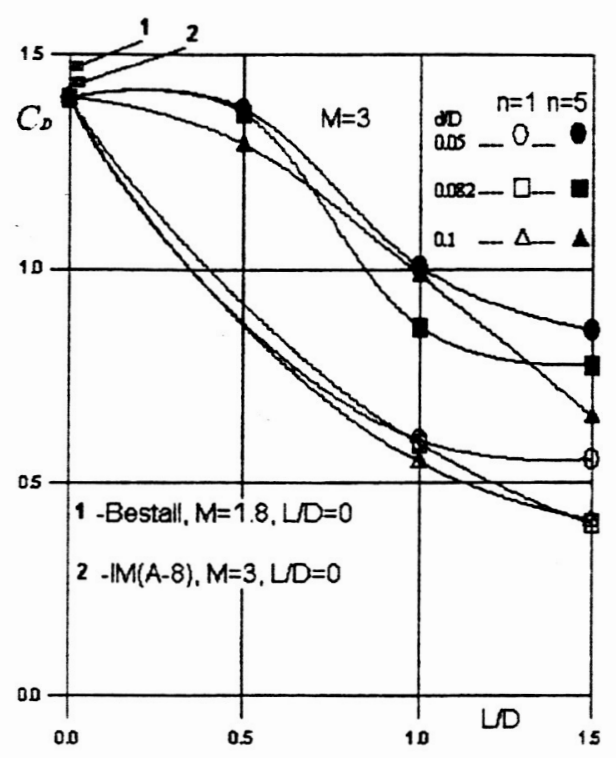

Fig.2 Drag coefficient, $C_{D}$, vs relative needle length, $\mathrm{L} / \mathrm{D}$, where $\mathrm{D}$-butt-end diameter, for single needle, $\mathrm{n}=1$, and 5 needles, $n=5$. Two experimental test results are shown for comparison: 1-obtained in Bestall experiments, and 2-obtained in IM/MSU transonic wind tunnel, A-8.
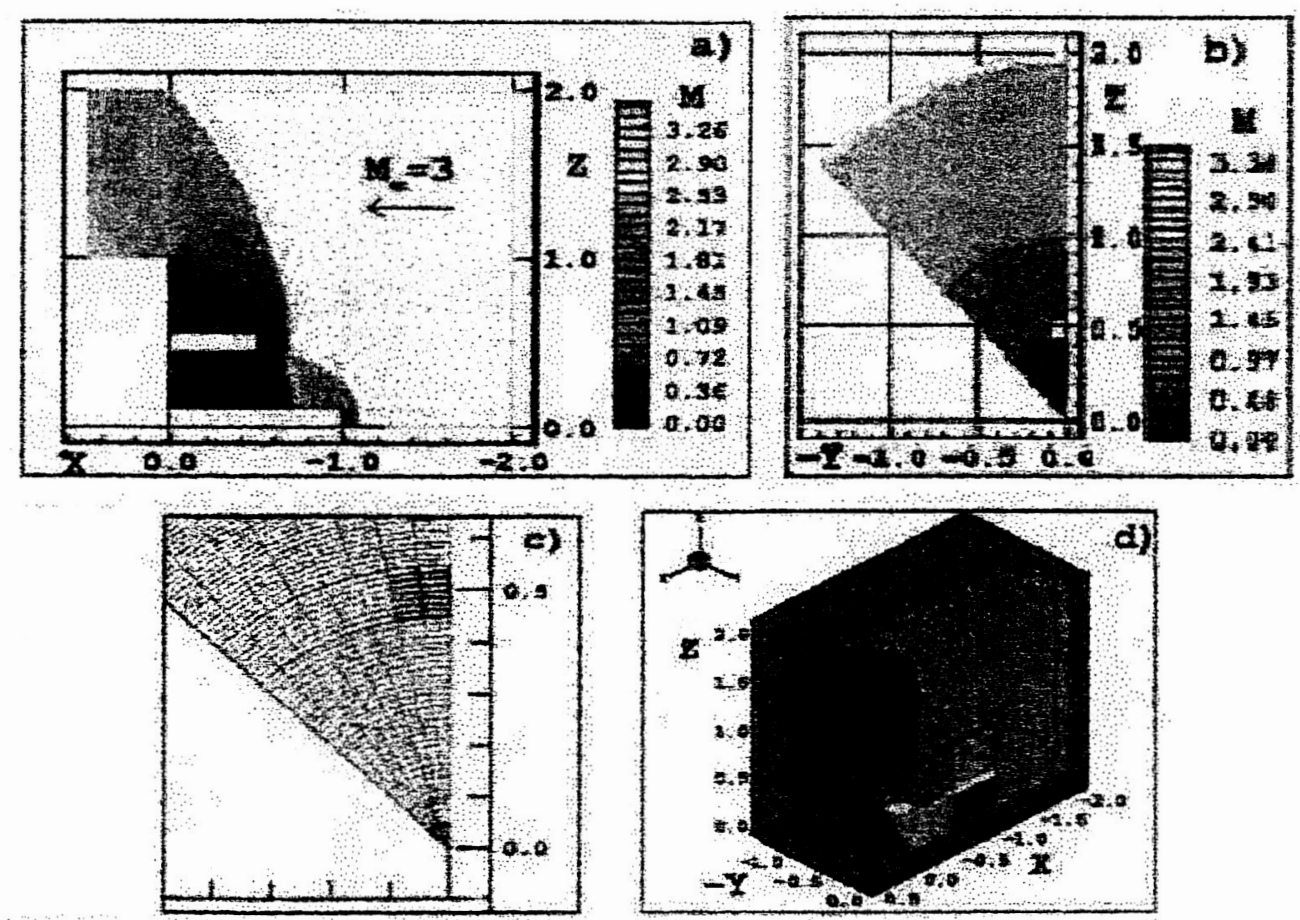

Fig.3 Mach 3 supersonic flow numerical simulation results around 5-needles nosed butt-end at zero attack angle. NASA CFL3D code. Navier-Stokes based simulation with slip boundary confitions. a) Mach contours in the symmetry XZ-plane; b) Mach contours in the cross section $X=$ const at the butt-end front wall; c) portion of employed grid in this cross section; d) 3D 
grid for the domain between two symmetry planes. This grid contents 15 blocks.
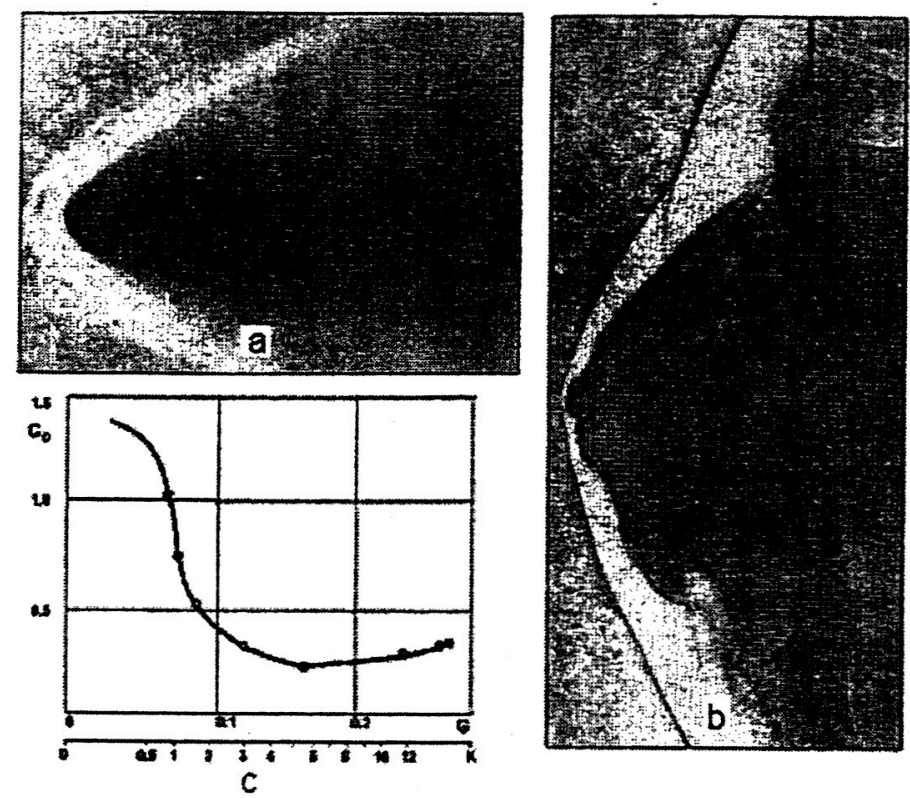

Fig.4 Experimental test results of liquid jet injection through a conical body and butt-end into the supersonic flow obtained in the IM/MSU aerodynamic wind tunnel A-7.

a) Liquid jet exhausted from conical body b) Liquid jet exhausted from butt-end

c) Drag coefficient, $C D$, versus impulse, $K$, and mass flow rate, $G$.
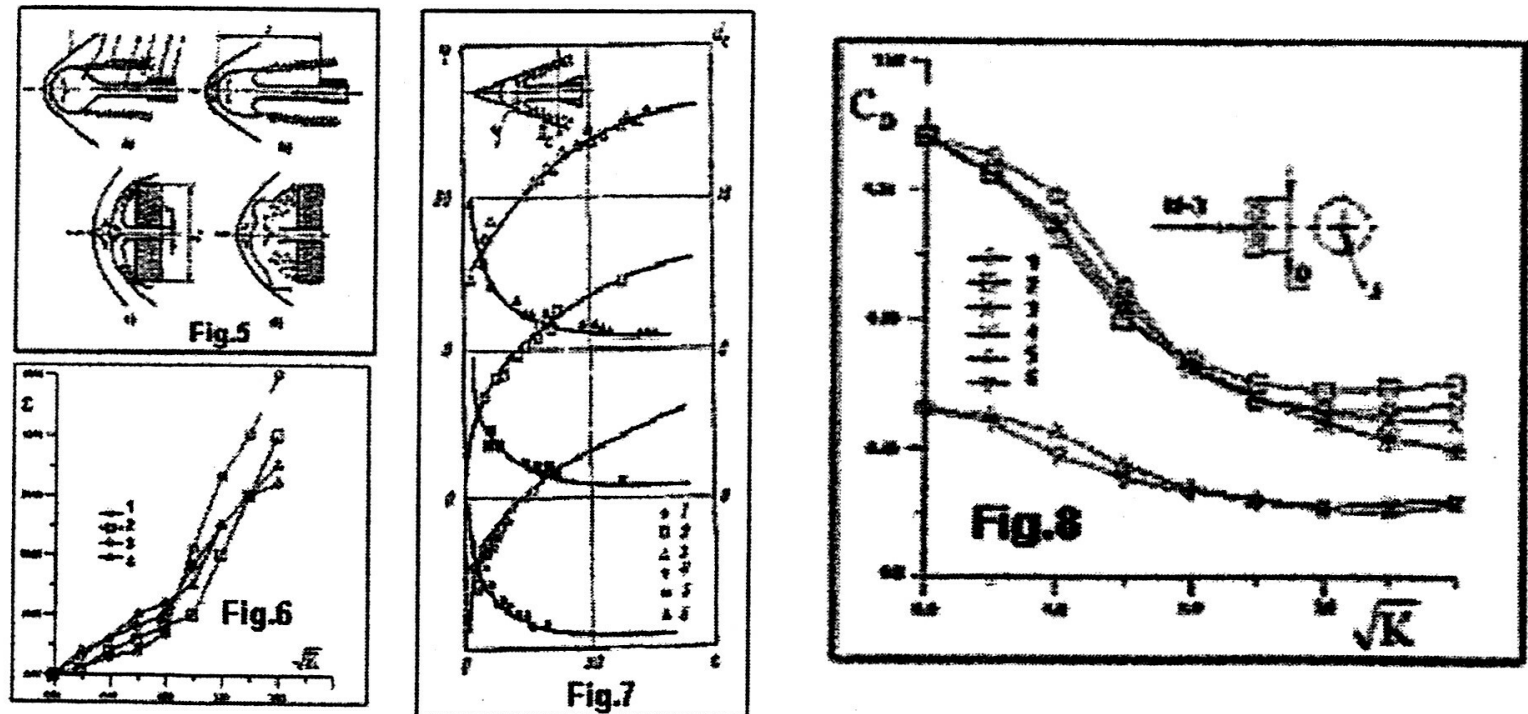

Fig.5 Possible 4 schemes of supersonic flow interaction with liquid jet exhausted from conical nozzle and butt-end observed in experiments:

a) Spherical-shaped jet; b) Mushroom-shaped jet; c) Two-layer stationary regime; d) Pulse unstable regime.

Fig.6 Liquid jet injection against the flow with Mach number, $M=3$. Dependence of the relative penetration depth, $\varepsilon=1 / \mathrm{r}$ and r-nozzle radius. $1-3$ are jets from a conical nozzle with $d=3,4$, and $5 \mathrm{~mm} ; 4$ is for jet from butt-end hole with $d=3 \mathrm{~mm}$

Fig.7 Dependence of conical jet angle, $\varphi$, and relative diameter, $d_{c}=D_{c} / d$, of relative jet depth penetration, $\varepsilon=1 / r$, where r-nozzle radius, $1-3$ are for $d_{c}=f(\varepsilon), d=3,4,5 \mathrm{~mm}$, and $4-6$ are for $\varphi=f(\varepsilon), d=3,4,5 \mathrm{~mm}$.

Fig.8 Drag coefficient, $C_{D}$, vs root of relative impulse coefficient, $\sqrt{\mathrm{K}}$. For liquid jet exhausted from: 1-butt-end $(\mathrm{d} / \mathrm{D}=0.05) ; 2$-butt-end $(\mathrm{d} / \mathrm{D}=0.025) ; 3$-butt-end with needle $(\mathrm{d} / \mathrm{D}=0.1) ; 4$-butt-end with needle $(\mathrm{d} / \mathrm{D}=0.05)$; 
5- semi-spherical body $(\mathrm{d} / \mathrm{D}=0.05)$; and 6-semi-spherical body $(\mathrm{d} / \mathrm{D}=0.025)$.

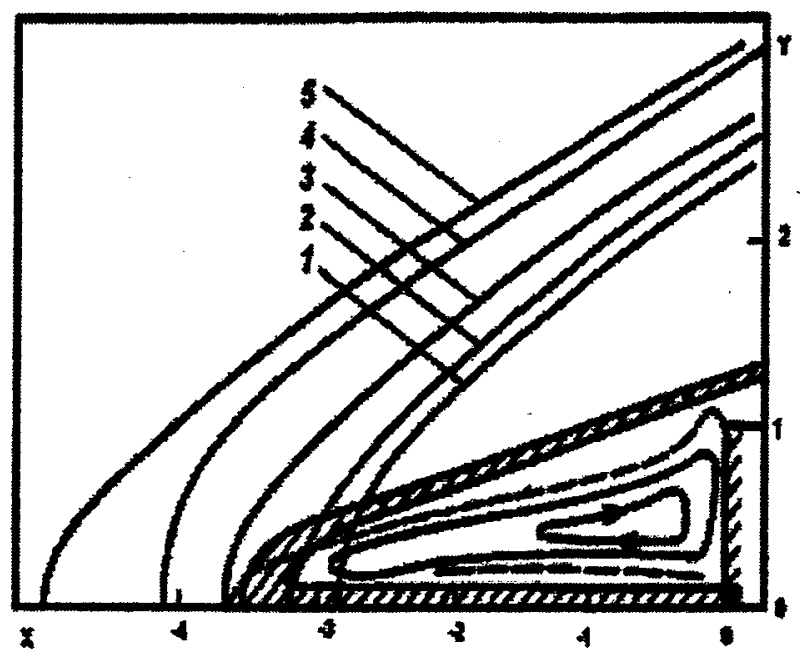

Fig.9 Geometric picture of two-phase jet (gas-solid particles) injection from the hole of the butt-end to the supersonic gas flow with Mach number, $M_{\infty}=3$. Numerical simulation results. Here are shown the bow shock waves, jet boundaries and several gas streamlines. 1- $\varepsilon=0 ; 2-\varepsilon=0.3, a_{\mathrm{p}}=0.5 \mu \mathrm{km} ; 3-\varepsilon=1, \mathrm{a}_{\mathrm{p}}=1 \mu \mathrm{km}$;

$5-\varepsilon=0.3, a_{p}=1.5 \mu \mathrm{km}$

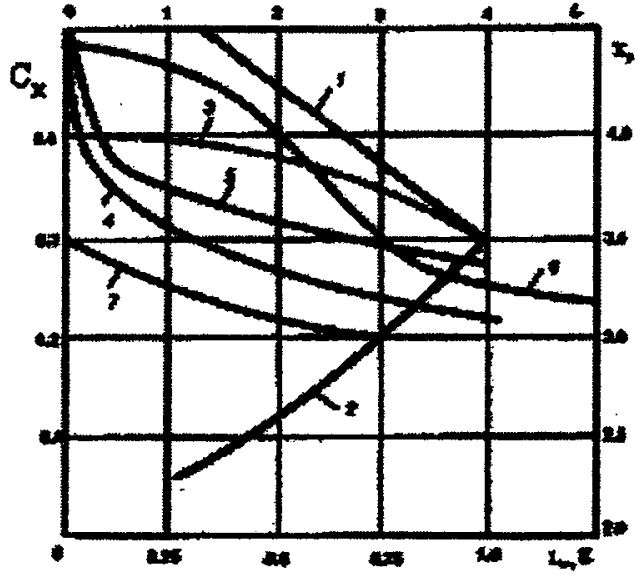

Fig.11 Dependence of wave drag coefficient of butt-end vs particle-gas lag coefficient, $L_{w}$, particle mass flow rate, $\varepsilon$, needle length, 1 , and jet force, $F$.

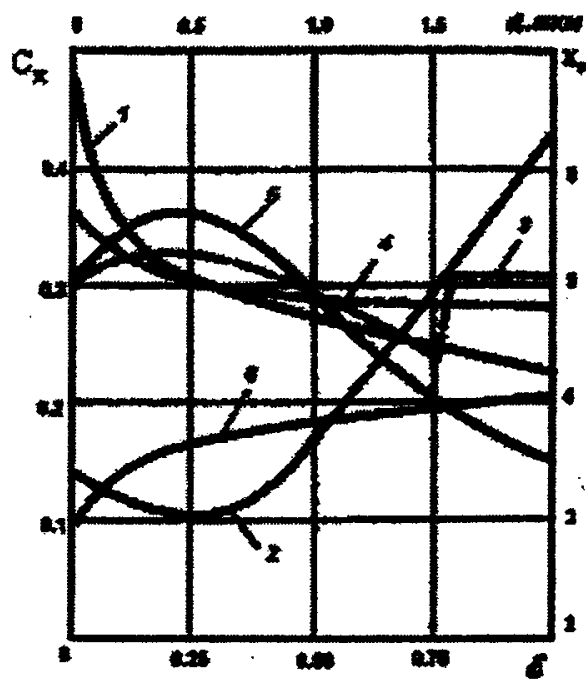

Fig.10 Dependence of drag coefficient, $\mathrm{C}_{\mathrm{xo}}$, and particle jet penetration depth of particle mass flow rate, $\varepsilon$, and particle radius, $a_{p}$.

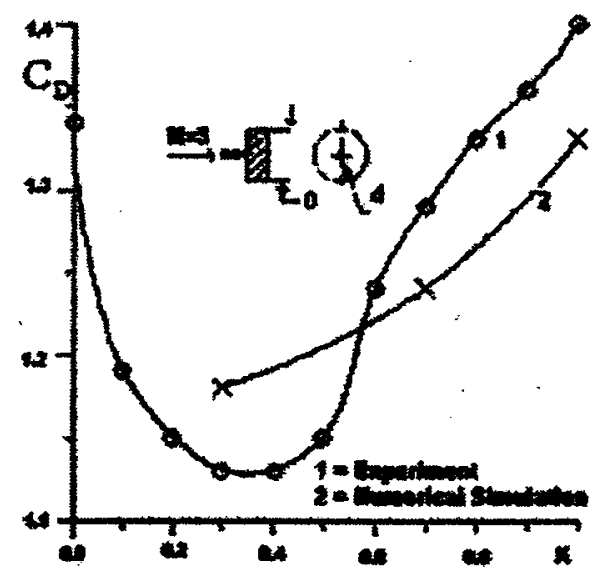

Fig.12 Comparison of butt-end drag coefficient, $C_{D}$, vs quarts particles jet impulse, $\mathrm{K}$, obtained in presented experimental and numerical simulation tests. These mmerical simulation results were obtained for particle size, $a_{p}=1 \mu \mathrm{km}$. 